\title{
Erratum to: A new look at 2D shallow water equations of fluid dynamics via multidimensional Kirchhoff paradigm
}

\author{
Sankar Basu' ${ }^{1}$. Alfred Fettweis ${ }^{2}$
}

Published online: 26 August 2015

(c) Springer Science+Business Media New York 2015

\section{Erratum to: Multidim Syst Sign Process DOI 10.1007/s11045-015-0321-z}

During the production process, typesetting errors were introduced. Please find the corrections below:

Equation number (3.9) refers to the entire group of equations expressing $\mathrm{L}_{0}, \mathrm{~L}_{1}, \mathrm{~L}_{2}, \mathrm{~L}_{3}$, and $\mathrm{L}_{4}$, and thus should have been aligned with the line above.

In Fig. 5 all T's are meant to be bold faced, i.e., $\mathrm{T}_{1}, \mathrm{~T}_{2}, \mathrm{~T}_{3}$, and $\mathrm{T}_{4}$ must be as $\mathbf{T}_{\mathbf{1}}, \mathbf{T}_{\mathbf{2}}, \mathbf{T}_{\mathbf{3}}$, and $\mathbf{T}_{\mathbf{4}}$ respectively.

In Fig. 7 all T's are meant to be bold faced, i.e., $\mathrm{T}_{1}, \mathrm{~T}_{2}$, and $\mathrm{T}_{3}$, must be as $\mathbf{T}_{\mathbf{1}}, \mathbf{T}_{\mathbf{2}}$, and $\mathbf{T}_{3}$ respectively.

The line after (7.12) should read:

and consequently with $a_{0}=\gamma_{1} a_{1}+\cdots+\gamma_{n-1} a_{n-1}$,

All equations in the Appendix have been numbered as (7.*). This makes it appear as though the Appendix is Section 7 of the paper. We meant the equations in the Appendix to have the format (A.*) instead. Thus, all equations in the paper of the type (7.*) should be replaced by corresponding equation numbers in the format (A.*).

Further comments provided by the authors:

For the paragraph before equation (3.26):

For the MD Kirchhoff circuit in Fig. 2 to be MD passive the MD inductances must have nonnegative values. These requirements enforce conditions on the parameters $\alpha$ and $v_{3}$ that

The online version of the original article can be found under doi:10.1007/s11045-015-0321-z.

Sankar Basu

sabasu@nsf.gov

1 Division of Computing and Communications Foundations, National Science Foundation, Arlington, VA 22230, USA

2 Lehrstuhl für Nachrichtentechnik, Ruhr-Universität Bochum, 44780 Bochum, Germany 
we have chosen according to $L_{0} \geq 4 / 3$ and $L_{\kappa} \geq 1$ for $\kappa=1$ to 3 . Since the two series inductances in Fig. 2, 1, $\mathrm{D}_{3}$ and $\mathrm{L}_{3}-1, \mathrm{D}_{3}$, can be combined into one inductance $\mathrm{L}_{3}, \mathrm{D}_{3}$, strictly speaking the requirements for MD passivity, and thus the global stability of the circuit is $L_{0} \geq 4 / 3, L_{\kappa} \geq 1$ for $\kappa=1$ to 2 and $L_{3} \geq 0$. However, the requirements $L_{0} \geq 4 / 3, L_{\kappa} \geq 1$ for $\kappa=1$ to 3 , as adopted, can be conveniently satisfied, and they suffice for our purpose.

\section{For the paragraph before equation (4.21):}

Likewise, in Fig. 4 the two series inductances $1, \mathrm{D}_{3}$ and $\hat{\mathrm{L}}_{3}-1, \mathrm{D}_{3}$ can be combined into one inductance $\hat{\mathrm{L}}_{3}, \mathrm{D}_{3}$. Once again, strictly speaking the requirements for the circuit in Fig. 4 to be MD passive are that $\hat{L}_{\kappa} \geq 1$ for $\kappa=1$ to $2, \hat{L}_{3} \geq 0$ and $\hat{L}_{\kappa 0} \geq 4 / 3$ for $\kappa=1$ to 3 . However, the requirements $\hat{L}_{\kappa} \geq 1$ for $\kappa=1$ to 3 , and $\hat{L}_{\kappa 0} \geq 4 / 3$ for $\kappa=1$ to 3 are sufficient for our purposes and have been used for a more streamlined treatment. 